MUCOSAL IMMUNOLOGY

\section{Acid attack}

Immune responses in the intestine must be tightly controlled to prevent harmful attacks against commensal organisms, food proteins and self antigens. Previous studies have shown that dendritic cells (DCs) contribute to immune homeostasis in the intestine by constitutively generating high levels of retinoic acid, which, together with transforming growth factor- $\beta$ (TGF $\beta$ ), promotes the development of regulatory $\mathrm{T}\left(\mathrm{T}_{\mathrm{Reg}}\right)$ cells. However, a recent paper in Nature exposes another side to retinoic acid, showing that this vitamin A metabolite can team up with interleukin-15 (IL-15) to drive pro-inflammatory responses against food antigens.

Patients with coeliac disease (who develop intestinal pathology owing to aberrant immune responses to dietary gluten) have increased levels of IL-15 in the gut mucosa, so DePaolo et al. explored whether this cytokine could affect the ability of intestinal DCs to promote $\mathrm{T}_{\mathrm{Reg}}$ cell conversion. Compared with untreated controls, IL-15-treated intestinal DCs were less efficient in driving $\mathrm{T}_{\text {Reg }}$ cell differentiation in vitro and, unexpectedly, the addition of retinoic acid to the IL-15-treated DCs further limited their capacity to induce $\mathrm{T}_{\mathrm{Reg}}$ cells. The authors extended these findings in vivo by using IL-15-transgenic mice that overexpress IL-15 in the intestinal lamina propria and mesenteric lymph nodes (MLNs). Feeding wild-type mice ovalbumin (OVA) led to the appearance of OVA-specific $\mathrm{T}_{\text {Reg }}$ cells in MLNs, but significantly fewer OVA-specific $\mathrm{T}_{\text {Reg }}$ cells were detected in the MLNs of OVA-fed IL-15-transgenic mice. Moreover, $\mathrm{T}_{\mathrm{Reg}}$ cell induction was further inhibited if IL-15-transgenic mice were fed retinoic acid together with OVA.

Using splenic DCs, which do not constitutively produce retinoic acid, the authors found that retinoic acid synergized with IL-15 to promote DC-mediated production of the proinflammatory cytokines IL-12p70 and IL-23. In keeping with this, intestinal lamina propria cells from OVAfed IL-15-transgenic mice, but not from wild-type controls, produced interferon- $\gamma(\mathrm{IFN} \gamma)$ in response to stimulation with OVA in vitro. This antigen-specific production of IFN $\gamma$ was enhanced if the IL-15-trangenic mice were fed retinoic acid along with OVA.

In a series of further experiments, the authors showed that retinoic acid could synergize with IL-15 and either

IL-12p70 or IL- 6 to promote the development of a T helper 1 $\left(\mathrm{T}_{\mathrm{H}} 1\right)$ - or $\mathrm{T}_{\mathrm{H}} 17$-type response, respectively. Exploration of the signalling mechanisms involved showed that retinoic acid and IL-15 synergized to promote pro-inflammatory DC responses in a JUN N-terminal kinase (JNK)-dependent manner.

Finally, DePaolo et al. addressed a question that has long puzzled immunologists. Although approximately $40 \%$ of the population express HLA-DQ2 and HLA-DQ8 (the MHC molecules that are associated with coeliac disease), only $2 \%$ of these individuals develop this disease. So what promotes the breakdown of tolerance to dietary gluten in this minority? The authors crossed IL-15-transgenic mice with HLA-DQ8-transgenic mice and found that the double-transgenic mice developed several hallmarks of early-stage coeliac disease in response to gluten feeding. These features included the development of gluten-specific antibodies and gluten-specific $\mathrm{T}_{\mathrm{H}} 1$ cells. Notably, the gluten-specific $\mathrm{T}_{\mathrm{H}} 1$ cell response was enhanced by retinoic acid, suggesting that in the presence of IL-15, retinoic acid can contribute to the breakdown of tolerance to food antigens.

This study suggests that retinoic acid is not simply an agent of tolerance, but synergizes with other cytokines present in the intestinal environment to potentiate both pro- and anti-inflammatory DC responses. In addition, the authors propose that in the HLA-DQ8 and IL-15 double-transgenic mice, immunologists may finally have a physiologically relevant mouse model of coeliac disease.

Yvonne Bordon

ORIGINAL RESEARCH PAPER DePaolo, R. W. et al. Co-adjuvant effects of retinoic acid and IL-15 induce inflammatory immunity to dietary antigens. Nature 10 Feb 2011 (doi:10.1038/nature09849) 